\title{
Global Stability of FAST TCP in Single-Link Single-Source Network
}

\author{
Joon-Young Choi, Kyungmo Koo, Jin S. Lee, and Steven H. Low
}

\begin{abstract}
We consider a single-link single-source network with FAST TCP source, and propose a static approximation of queuing delay dynamics at the link. The static approximation turns out to be a form with network feedback delay, which enables to analyze FAST TCP reflecting the effect of network feedback delay. Based on a continuous-time dynamic model of FAST TCP, we achieve the boundedness of window size and a sufficient condition for global asymptotic stability. The simulation results illustrate the validity of the sufficient condition for global asymptotic stability.
\end{abstract}

\section{INTRODUCTION}

Commonly implemented transmission control protocols (TCP) in the current Internet such as TCP Reno and its variants control their congestion window size based on the well-known additive increase multiplicative decrease (AIMD) mechanism [1] [2]. However, it is shown that these algorithms are not scalable as the delay-bandwidth product of the network becomes larger [3] [4] [5] because additive increasing is too slow and multiplicative decreasing is too severe in the large delay-bandwidth product network.

To cope with this problem, several congestion control algorithms have been proposed for high speed networks: HSTCP [6], STCP [7], FAST TCP [5], and BIC TCP [8]. FAST TCP has a feature that the queuing delay is used as a congestion measure. While the packet loss that is used as a congestion measure in TCP Reno has only binary information about the congestion, the queuing delay indicates a level of congestion, which means how far the current state is from the equilibrium [5]. Hence, the congestion control mechanism developed using queuing delay as a congestion measure is more responsive to the network congestion and, if the algorithm is stable, makes the network state be always around the equilibrium [5].

Even though extensive experiments of FAST TCP have been conducted and the results are promising [5], stability property of FAST TCP has not been sufficiently studied yet. Local stability of FAST TCP without network feedback delay was proved for the case of a single link in [5]. A sufficient condition for local asymptotic stability of FAST TCP was achieved for the general network with network feedback delay in [9]. However, there is no analysis on global stability of FAST TCP in the presence of the network feedback delay.

In this paper, we investigate the global stability of singlelink single-source network with FAST TCP source in the

J.-Y. Choi is with the Department of Electronic Engineering, $\mathrm{Pu}$ san National University, Korea; Email: jyc@pusan.ac.kr. K. Koo and J. S. Lee are with the Department of Electronic and Electrical Engineering, Pohang University of Science and Technology, Korea; Email: \{pumpkins,jsoo\}@postech.ac.kr. S. H. Low is with the Departments of Computer Science and Electrical Engineering, California Institute of Technology; Email: slow@caltech.edu.

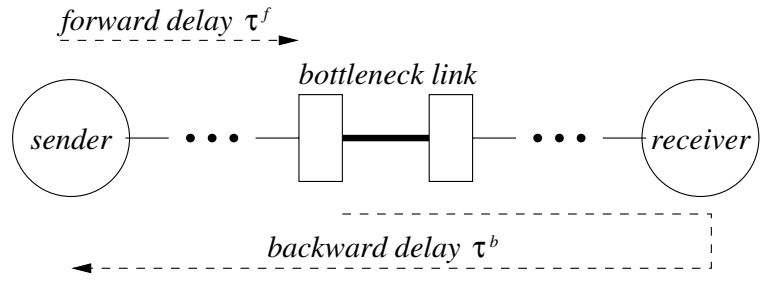

Fig. 1. Network model with delays

presence of the network feedback delay. We propose a static approximation of queuing delay dynamics, which turns out to properly describe the feedback delay between window size and queuing delay measured at the source. Based on a continuous-time model of FAST TCP and the static approximation of the queuing delay model, we establish a sufficient condition for global asymptotic stability of FAST TCP by adopting a Lyapunov-Krasovskii functional. Moreover, the condition is improved by taking advantage of a change of state variables.

This paper is organized as follows. Section II describes the single-link single-source network model of FAST TCP. Section III analyzes the global boundedness property of FAST TCP. Section IV presents a sufficient condition for global asymptotic stability of FAST TCP. Section V provides simulation results and discussions. Section VI makes conclusions.

\section{Network MOdel}

Since analyzing a general multi-link multi-source network with TCP sources considering global stability causes mathematical difficulty and complexity, we focus on the case of a single-link single-source network in which a pair of sender and receiver node is connected through a single bottleneck link as depicted in Fig. 1. The link has a finite transmission capacity $c$ and is assumed to have infinite buffering storage. Associated with the link is the queuing delay $p(t)$ and with the source is the source rate $x(t)$. We assume at time $t$ that the source observes as a feedback signal the aggregated queuing delay in its path

$$
q(t):=p\left(t-\tau^{b}\right),
$$

where $\tau^{b}$ denotes the backward delay in the feedback path from link to source, and the link observes the aggregated source rate

$$
y(t):=x\left(t-\tau^{f}\right),
$$

where $\tau^{f}$ denotes the forward delay from source to link. The round trip time (RTT) $T(t)$ is defined for the source 
as $T(t):=d+q(t)$, where $d$ is the constant round trip propagation time, and the round trip feedback delay is assumed to be $T(t):=\tau^{f}+\tau^{b}$.

A model of FAST TCP with its associated queue management was presented in [9] as a continuous-time dynamic system:

$$
\begin{aligned}
\dot{w}(t) & =\gamma\left(\frac{d w(t)}{d+q(t)}+\alpha-w(t)\right) \\
\dot{p}(t) & =\frac{1}{c}(y(t)-c),
\end{aligned}
$$

where $w(t)$ is the congestion window size of the source, $\alpha>0$ and $\gamma \in(0,1]$ are the congestion control parameters, and the time is measured in the unit of update period in FAST TCP. Note that in [9] the source rate is defined at time $t$ in terms of $w(t)$ as

$$
x(t):=\frac{w(t)}{d+q(t)} .
$$

Based on the self-clocking property of TCP [2] and ignoring the fast dynamics at the link, a static approximation of the dynamic model of queuing delay was proposed in [9] for a discrete-time FAST TCP model:

$$
\frac{w\left(t-\tau^{f}\right)}{d+q\left(t-\tau^{f}\right)}\left\{\begin{array}{l}
=c \text { if } p(t)>0 \\
\leq c \text { if } p(t)=0
\end{array}\right.
$$

Since this static equation does not describe any feedback delay between $w(t)$ and $q(t)$, it is not appropriate for the continuous-time model of FAST TCP. To capture the effect of the network feedback delay, we propose a static approximation of queuing delay dynamics as

$$
p(t)=\left[\frac{w\left(t-\tau^{f}\right)}{c}-d\right]^{+},
$$

where $[x]^{+}:=\max \{0, x\}$, and the feedback delay between $w(t)$ and $q(t)$ is obviously represented differently from (5). The static model of queuing delay given by (6) can be interpreted as the queuing delay at the link is algebraically determined by the delayed window size of the source. That is, in the static model of queuing delay, the window size and queuing delay are regarded as the input and output rather than the source sending rate and queuing delay as in (5). This approach is more reasonable for the windowbased congestion control algorithm such as FAST TCP. In addition, the static queuing delay model (6) can account for the oscillation phenomenon of source window size, which is well known to be mainly due to the network feedback delay. A variety of experiment results for FAST TCP are found in [10], and oscillations of window size of FAST TCP are observed in some of the results.

Using (1) and (6), we get the aggregate queuing delay that is measured at the source as

$$
\begin{aligned}
q(t) & =p\left(t-\tau^{b}\right) \\
& =\left[\frac{w\left(t-\tau^{f}-\tau^{b}\right)}{c}-d\right]^{+} \\
& =\left[\frac{w(t-T(t))}{c}-d\right]^{+},
\end{aligned}
$$

from which we can define the source rate as

$$
x(t):=\frac{w(t-T(t))}{d+q(t)}=c .
$$

Note that the static model of queuing delay (6) reflect both the self-clocking property and the feedback delay between window size and queuing delay measured at the source. Substituting (7) into (3), we obtain the whole closed-loop system as

$$
\dot{w}(t)=\gamma \alpha
$$

for $w(t-T(t)) \leqslant c d$, and

$$
\dot{w}(t)=\gamma\left(\frac{c d}{w(t-T(t))} w(t)+\alpha-w(t)\right)
$$

for $w(t-T(t))>c d$, which is a nonlinear delay differential equation. The corresponding equilibrium point $w^{*}$ of (8) and (9) is uniquely determined as $w^{*}=\alpha+c d>0$.

\section{BOUNDEDNESS}

In this section, we investigate the ultimate boundedness properties of $w(t)$ with respect to time $t$. We now show in the following lemma that $w(t)$ is ultimately bounded below.

Lemma 1: There exists a $T_{L}>0$ such that

$$
w(t) \geq \alpha \text { for all } t>T_{L} .
$$

Proof: From (9), we obtain the following inequality:

$$
\dot{w}(t) \geq \gamma(\alpha-w(t))
$$

from which we have $w(t) \geq \alpha$ for all $t>T_{L}$, where $T_{L}$ is a sufficiently large time value.

The following lemma shows that $w(t)$ and $q(t)$ is ultimately bounded above.

Lemma 2: $w(t)$ and $q(t)$ are uniformly bounded above.

Proof: Whenever $w(t-T(t))>c d$, it holds that

$$
\dot{w}(t)=\gamma(-k w(t)+\alpha),
$$

where $0<k<1$ from (9). Hence, $w(t)$ is bounded above, and consequently $q(t)$ is also bounded above from (7).

In the subsequent analysis, we fix $T(t)$ as a constant $T$ defined as $T:=\max T(t)$, where the existence of $T$ is guaranteed by Lemma 2 because $T(t)=d+q(t)$.

\section{Global Asymptotic Stability}

In this section, we establish a sufficient condition for global asymptotic stability of (9).

\section{A. Lyapunov-Krasovskii functional}

For facility of analysis, we make a change of variable in terms of the window size error $\tilde{w}(t):=w(t)-w^{*}=$ $w(t)-\alpha-c d$ in (9):

$$
\dot{w}(t)=\gamma\left(\frac{c d}{w(t-T)}(\tilde{w}(t)-\tilde{w}(t-T))-\tilde{w}(t)\right)
$$

Theorem 1: If $\alpha>2 c d$, then the FAST TCP described by (9) is globally asymptotically stable. 
Proof: Define a Lyapunov-Krasovskii functional as

$$
V(w(t), t)=\frac{1}{2} w^{2}(t)+\frac{1}{2} \gamma c d \int_{t-T}^{t} \frac{\tilde{w}^{2}(\theta)}{w(\theta)} d \theta,
$$

then from Lemma 1, we have

$$
\begin{aligned}
& \dot{V}(w(t), t) \\
\leq & \gamma\left(\frac{c d}{w(t-T)}\left(\tilde{w}^{2}(t)-\tilde{w}(t-T) \tilde{w}(t)\right)-\tilde{w}^{2}(t)\right) \\
& +\frac{\gamma c d}{2}\left(\frac{\tilde{w}^{2}(t)}{w(t)}-\frac{\tilde{w}^{2}(t-T)}{w(t-T)}\right) \\
= & \frac{\gamma c d}{w(t-T)} \tilde{w}^{2}(t)-\gamma c d \frac{\tilde{w}(t-T)}{w(t-T)} \tilde{w}(t)-\gamma \tilde{w}^{2}(t) \\
& +\frac{\gamma c d}{2} \frac{\tilde{w}^{2}(t)}{w(t)}-\frac{\gamma c d}{2} \frac{\tilde{w}^{2}(t-T)}{w(t-T)} \\
\leq & \frac{\gamma c d}{w(t-T)} \tilde{w}^{2}(t)+\frac{\gamma c d}{2} \frac{\tilde{w}^{2}(t-T)}{w(t-T)}+\frac{\gamma c d}{2 w(t-T)} \tilde{w}^{2}(t) \\
& -\gamma \tilde{w}^{2}(t)+\frac{\gamma c d}{2} \frac{\tilde{w}^{2}(t)}{w(t)}-\frac{\gamma c d}{2} \frac{\tilde{w}^{2}(t-T)}{w(t-T)}
\end{aligned}
$$

(from Young's inequality)

$$
\begin{aligned}
= & \frac{\gamma c d}{w(t-T)} \tilde{w}^{2}(t)+\frac{\gamma c d}{2 w(t-T)} \tilde{w}^{2}(t) \\
& -\gamma \tilde{w}^{2}(t)+\frac{\gamma c d}{2} \frac{\tilde{w}^{2}(t)}{w(t)} \\
\leq & -\gamma\left(\frac{\alpha-2 c d}{\alpha}\right) \tilde{w}^{2}(t) \text { for all } t>T_{L},
\end{aligned}
$$

where $T_{L}$ is a sufficiently large time value. Then, according to Theorem 2.1 of Chapter 4 in [11], the theorem is proved.

\section{B. Improvement of the condition}

We improve the sufficient condition presented in the previous subsection by taking advantage of the specific property FAST TCP (9) and making a change of variable as

$$
z(t):=\frac{1}{w(t)} .
$$

Then, the derivative of $z(t)$ is calculated from (9) as

$$
\begin{aligned}
\dot{z}(t) & =\frac{1}{w(t)}-\frac{c d}{w(t-T) w(t)}-\frac{\alpha}{w^{2}(t)} \\
& =z(1-c d z(t-T)-\alpha z(t)) \\
& =-z(c d \tilde{z}(t-T)+\alpha \tilde{z}(t)),
\end{aligned}
$$

where $\tilde{z}(t):=z(t)-z^{*}=z(t)-\frac{1}{\alpha+c d}$. Now, making another change of variable as

$$
u(t):=\ln z(t) \text { i.e. } z(t)=e^{u(t)},
$$

we obtain the dynamic equation of $u(t)$ as

$$
\dot{u}(t)=-\frac{c d}{\alpha+c d}\left(e^{\tilde{u}(t-T)}-1\right)-\frac{\alpha}{\alpha+c d}\left(e^{\tilde{u}(t)}-1\right),
$$

where $\tilde{u}(t):=u(t)-u^{*}=u(t)-\ln \frac{1}{\alpha+c d}$. We present an improved sufficient condition for global asymptotic stability of FAST TCP (9) in the following theorem.

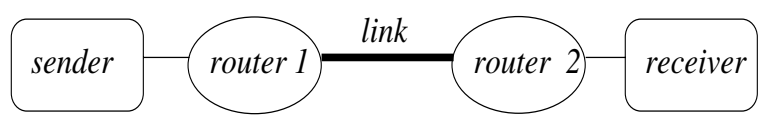

Fig. 2. Network topology

Theorem 2: If $\alpha>c d$, then the FAST TCP described by (9) is globally asymptotically stable.

Proof: Define a Lyapunov-like functional

$$
V(\tilde{u}(t), t)=|\tilde{u}(t)|+\frac{c d}{\alpha+c d} \int_{t-T}^{t}\left|e^{\tilde{u}(\theta)}-1\right| d \theta,
$$

and considering the upper right-hand derivative of $V(\tilde{u}(t), t)$ along the solution of (12), we have

$$
\begin{aligned}
& D^{+} V(\tilde{u}(t), t) \\
= & \dot{\tilde{u}}(t) \operatorname{sgn}(\tilde{u}(t))+\frac{c d}{\alpha+c d}\left(\left|e^{\tilde{u}(t)}-1\right|-\left|e^{\tilde{u}(t-T)}-1\right|\right) \\
= & -\frac{c d}{\alpha+c d}\left(e^{\tilde{u}(t-T)}-1\right) \operatorname{sgn}(\tilde{u}(t)) \\
& -\frac{\alpha}{\alpha+c d}\left(e^{\tilde{u}(t)}-1\right) \operatorname{sgn}(\tilde{u}(t)) \\
& +\frac{c d}{\alpha+c d}\left(\left|e^{\tilde{u}(t)}-1\right|-\left|e^{\tilde{u}(t-T)}-1\right|\right) \\
\leq & \left.\frac{c d}{\alpha+c d} \mid e^{\tilde{u}(t-T)}-1\right)\left|-\frac{\alpha}{\alpha+c d}\right|\left(e^{\tilde{u}(t)}-1\right) \mid \\
& +\frac{c d}{\alpha+c d}\left(\left|e^{\tilde{u}(t)}-1\right|-\left|e^{\tilde{u}(t-T)}-1\right|\right) \\
= & -\frac{\alpha-c d}{\alpha+c d}\left|e^{\tilde{u}(t)}-1\right|
\end{aligned}
$$

Since $\left|e^{\tilde{u}(t)}-1\right|>0$ whenever $\tilde{u}(t) \neq 0$, we can conclude from the Lyapunov Theorem that the solution of (12) is globally asymptotically stable [12].

Note that Theorem 2 is proved without the property of Lemma 1 while it is necessary to use the property of Lemma 1 in proving Theorem 1.

From the Theorem 1 and 2, we can conclude that a singlelink single-source network with FAST TCP source is globally asymptotically stable when the source parameter $\alpha$ is sufficiently large compared to the capacity-propagation delay product, $c d$ of the link. This condition can be satisfied in a decentralized way because each source can independently tune its own parameter $\alpha$ based on its link status. Note that, however, the larger $\alpha$ is, the larger queuing delay is, since the equilibrium queuing delay depends on $\alpha$ such that $q^{*}=\frac{\alpha}{c}$.

\section{Simulation}

In this section, we present a set of MATLAB simulation results to illustrate the validity of the sufficient condition for global asymptotic stability of FAST TCP presented in Theorem 2.

We conduct the simulation for a single bottleneck link utilized by a source with the topology shown in Fig. 2. We simulate three cases of networks with capacity $c$ and round trip latency between routers $d$ as $c=10 \mathrm{pkts} / \mathrm{ms}, d=10 \mathrm{~ms}$; $c=100 \mathrm{pkts} / \mathrm{ms}, d=10 \mathrm{~ms} ;$ and $c=50 \mathrm{pkts} / \mathrm{ms}, d=20 \mathrm{~ms}$, 
TABLE I

EQUILIBRIUM POINTS

\begin{tabular}{|c|c|c|c|}
\hline $\begin{array}{c}c(\mathrm{pkts} / \mathrm{ms}) \\
/ d(\mathrm{~ms})\end{array}$ & $\alpha$ (pkts) & $\begin{array}{c}\text { Window size } \\
(\mathrm{pkts})\end{array}$ & $\begin{array}{c}\text { Queuing delay } \\
(\mathrm{ms})\end{array}$ \\
\hline \multirow{2}{*}{$10 / 10$} & 80 & 180 & 8 \\
\cline { 2 - 4 } & 100 & 200 & 10 \\
\hline \hline \multirow{2}{*}{$100 / 10$} & 850 & 1850 & 8.5 \\
\cline { 2 - 4 } & 1000 & 2000 & 10 \\
\hline \multirow{2}{*}{$50 / 20$} & 900 & 1900 & 18 \\
\cline { 2 - 4 } & 1000 & 2000 & 20 \\
\hline
\end{tabular}

respectively. We set the congestion control parameter $\alpha$ (pkts) as indicated in Fig. 3, 4, and 5.

Table 1 summarizes the calculated equilibrium point of the source for each parameter, and Fig. 3, 4, and 5 show the simulation results for each network. All figures illustrate that window sizes are ultimately bounded, and window sizes asymptotically converge to their equilibrium points provided that $\alpha>c d$.

Moreover, as shown Fig. 3, 4, and 5, TCP FAST is not asymptotically stable and oscillatory with the parameter of $\alpha$ even slightly smaller than $c d$. This observation implies that the sufficient condition presented in Theorem 2 is not so conservative.

\section{CONCLUSIONS}

In this paper, we have analyzed the global asymptotic stability of FAST TCP in a single-link single-source network with feedback delay. We adopt a continuous-time source model of FAST TCP and a queue model approximated in a static manner, which is considered as the self-clocking property of TCP and includes network feedback delays explicitly. Based on this network model, we have derived a sufficient condition for global asymptotic stability, and this condition is verified by simulation experiments with various parameter conditions.

The sufficient condition for global asymptotic stability states that the source parameter $\alpha$ should be sufficiently large compared to the capacity-propagation delay product of the link. This condition can be satisfied in a decentralized way because each source can independently tune its own parameter based on its link status. However, since the large $\alpha$ induces large queuing delay, it needs to be adjusted adequately.

The results in this paper is limited to a simple case of single-link single-source network, but it is possible to extend to more general networks in future works.

\section{REFERENCES}

[1] Raj Jain, K. K. Ramakrishnan and Dah-Ming Chiu, "Congestion Avoidance in Computer Networks with a Connectionless Network Layer," Technical Report DEC-TR-506, Digital Equipment Corporation, August 1987.

[2] V. Jacobson, "Congestion Avoidance and Control," In Proceedings of ACM SIGCOMM'88, August 1988.

[3] C. V. Hollot, V. Misra, D. Towsley, and W. B. Gong, "Analysis and Design of Controllers for AQM Routers Supporting TCP Flows," IEEE Transactions on Automatic Control, Vol. 47, No. 6, pp. 945-959, 2002.

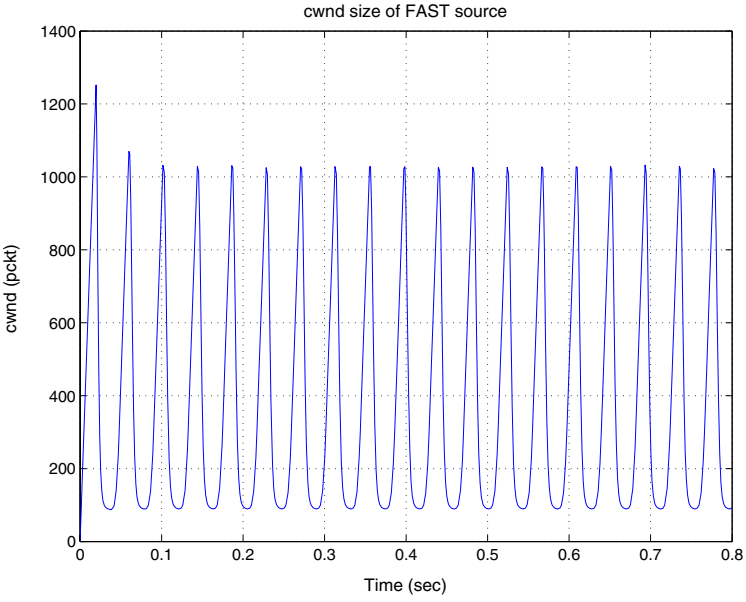

(a) $\alpha=80 \mathrm{pkts}$

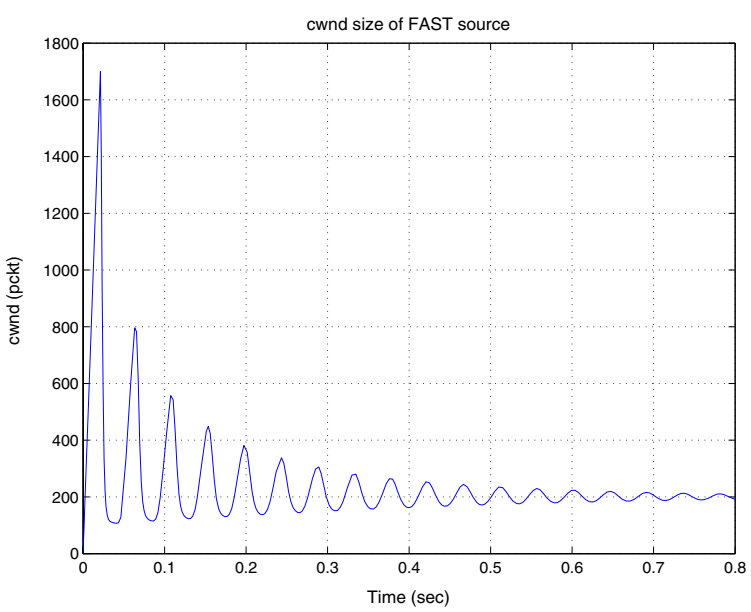

(b) $\alpha=100$ pkts

Fig. 3. Window size when $c=10 \mathrm{pkts} / \mathrm{ms}$ and $d=10 \mathrm{~ms}$

[4] Steven H. Low, Fernando Paganini, Jiantao Wang and John C. Doyle, "Linear Stability of TCP/RED and a Scalable Control," Computer Networks Journal, Vol. 43, No. 5, pp. 633-647, December 2003.

[5] Cheng Jin, David X. Wei and Steven H. Low, "FAST TCP: motivation, architecture, algorithms, performance," In Proceedings of IEEE Infocom, March 2004. http: //netlab.caltech. edu/FAST/

[6] Sally Floyd, "HighSpeed TCP for Large Congestion Windows," RFC 3649, Experimental, December 2003. http: / /www.icir.org/floyd/hstcp.html

[7] Tom Kelly, "Scalable TCP: Improving Performance in High-speed Wide Area Networks," Submitted for publication. http: / / www-lce.eng.cam.ac.uk/ ctk21/scalable/

[8] Lisong $\mathrm{Xu}$, Khaled Harfoush and Injong Rhee, "Binary Increase Congestion Control for Fast Long-distance Networks," In Proceedings of IEEE Infocom, March 2004.

[9] Jiantao Wang, David X. Wei and Steven H. Low, "Modelling and Stability of FAST TCP," In Proceedings of IEEE Infocom, April 2005.

[10] High-Speed Network Protocol Experiments. http: //netsrv.csc.ncsu.edu

[11] Jack K. Hale and Sjoerd M. Verduyn Lunel, Introduction to Functional Differential Equations, 3rd ed., Prentice Hall, 2002.

[12] Xue-zhong He, "Stability and Delays in a Predator-prey System," Journal of Mathematical Analysis and Applications, Vol. 198, pp. 355-370, 1996. 


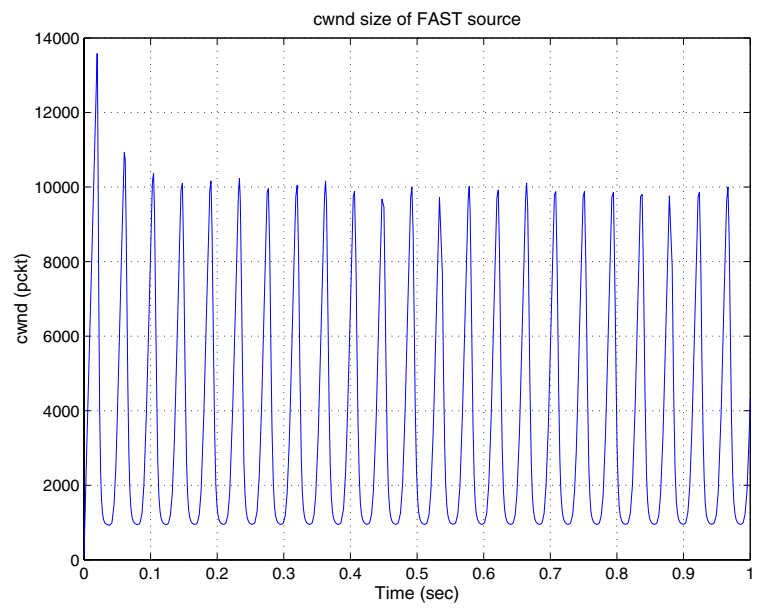

(a) $\alpha=850$ pkts

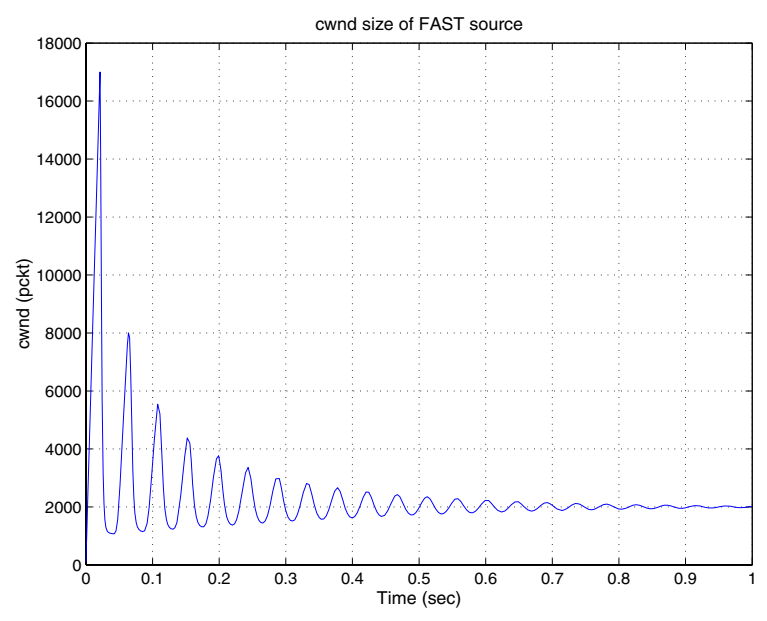

(b) $\alpha=1000$ pkts

Fig. 4. Window size when $c=100$ pkts $/ \mathrm{ms}$ and $d=10 \mathrm{~ms}$

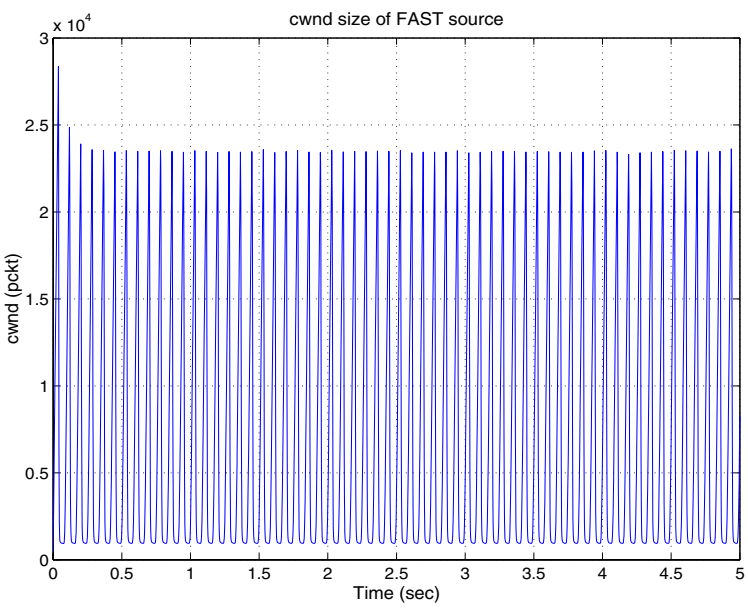

(a) $\alpha=900$ pkts

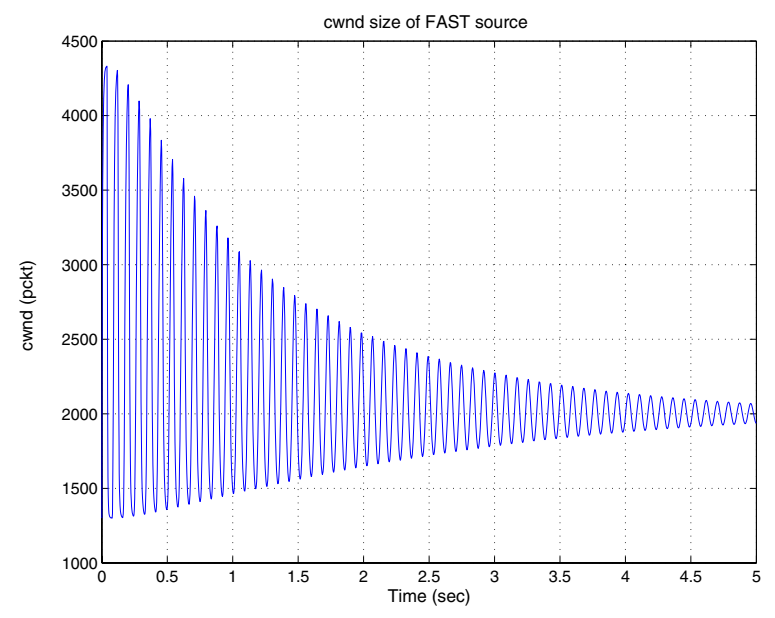

(b) $\alpha=1000$ pkts

Fig. 5. Window size when $c=50$ pkts $/ \mathrm{ms}$ and $d=20 \mathrm{~ms}$ 